\title{
Mütter-Screening in einem COVID-19-Niedrig-Pandemiegebiet: Bestimmung SARS-CoV-2-spezifischer Antikörper bei 401 Rostocker Müttern mittels ELISA und Immunfluoreszenz-Bestätigungstest

\author{
Screening of Mothers in a COVID-19 Low-Prevalence Region:
} Determination of SARS-CoV-2 Antibodies in 401 Mothers from Rostock by ELISA and Confirmation by Immunofluorescence
}

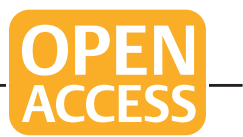

Autoren

Emil C. Reisinger ${ }^{1}$, Ronald von Possel ${ }^{2}$, Philipp Warnke ${ }^{3}$, Hilte F. Geerdes-Fenge ${ }^{1}$, Christoph J. Hemmer ${ }^{1}$, Susanne Pfefferle ${ }^{4}$, Micha Löbermann', Martina Littmann ${ }^{5}$, Petra Emmerich ${ }^{1,2}$

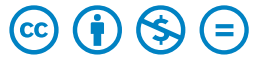

Institute

1 Abteilung für Tropenmedizin und Infektionskrankheiten, Klinik für Innere Medizin, Universitätsmedizin Rostock

2 Bernhard-Nocht-Institut für Tropenmedizin, Abteilung Virologie, Hamburg

3 Institut für Medizinische Mikrobiologie, Virologie und Hygiene, Universitätsmedizin Rostock

4 Institut für Medizinische Mikrobiologie, Virologie und Hygiene, Universitätsklinikum Eppendorf, Hamburg

5 Landesamt für Gesundheit und Soziales, MecklenburgVorpommern

Schlüsselwörter

SARS-CoV-2, COVID-19, Mütter, Kinder, Antikörperprävalenz

Key Words

SARS-CoV-2, COVID-19, mothers, children, antibody

prevalence

Bibliografie

DOI https://doi.org/10.1055/a-1197-4293

Online-Publikation: 22.6.2020

Dtsch Med Wochenschr 2020; 145: e96-e100

(c) Georg Thieme Verlag KG, Stuttgart · New York

ISSN 0012-0472

Korrespondenzadresse

Prof. Dr. Emil C. Reisinger, MBA

Abteilung für Tropenmedizin und Infektionskrankheiten

Klinik für Innere Medizin

Universitätsmedizin Rostock, Ernst-Heydemann-Straße 6,

D-18055 Rostock

Tel.: ++ 49/3 81/4947511

Fax: $++49 / 381 / 4947509$

emil.reisinger@uni-rostock.de

\section{ZUSAMMENFASSUNG}

Hintergrund Bei Kindern verläuft die Infektion mit SARSCoV-2, dem Erreger von COVID-19, in der Regel weniger schwer und häufiger symptomlos als bei Erwachsenen. Kinder können aber diese Infektion u. a. auf vulnerable Erwachsene übertragen. Daher sind zur Zeit Einrichtungen der Kinderbetreuung und Schulen teilweise geschlossen.

Methoden Da sich der Infektionsstatus bei Kindern unter 13 Jahren und deren Müttern meist ähnlich darstellt, untersuchten wir bei 401 Rostocker Müttern von Kindern zwischen 1 und 10 Jahren Rachenabstriche auf SARS-CoV-2 und Serumproben auf Antikörper gegen diesen Erreger.

Ergebnisse Wir fanden bei keiner der untersuchten Mütter RNA von SARS-CoV-2 im Rachenabstrich. Im ELISA waren IgG-Antikörper in einem Serum positiv, IgA-Antikörper waren in 11 Seren positiv und in 3 Seren grenzwertig. Alle 401 Seren waren im Fluoreszenz-Bestätigungstest (IIFT) mit FITC-markierten IgG-, IgA- und IgM-Antikörpern negativ.

Schlussfolgerung Im Untersuchungszeitraum waren bei den getesteten Rostocker Müttern weder RNA von SARS-CoV-2 noch spezifische Antikörper nachweisbar.

\section{ABSTRACT}

Background In children, the infection with SARS-CoV-2, the cause of COVID-19, tends to be clinically inapparent more often or less severe than in adults. The spread of this infection from children poses a danger to vulnerable adults. Therefore, child care institutions and schools currently are widely closed. Methods Since the status of infection tends to be congruent in mothers and their children, we tested 401 mothers of children between 1 and 10 years in the city of Rostock (State of Mecklenburg-Westpomerania, northeast of Germany), for the presence of RNA of SARS-CoV-2 in throat swabs, and of antibodies against SARS-CoV-2 in serum.

Results In none of the mothers tested, RNA of this virus was detected in the throat swab. In the ELISA test, IgG antibodies were positive in one serum sample, IgA antibodies were posi- 
tive in 11, and borderline in 3 serum samples. All 401 sera were negative in the indirect immunofluorescence test (IIFT) with FITC-labeled $\lg G$, IgA, und IgM antibodies.
Conclusion At the time of this study, neither SARS-CoV-2 RNA, nor specific antibodies against SARS-CoV-2 were detectable in the mothers tested in Rostock.

\section{Einleitung}

In allen deutschen Bundesländern wurden im März 2020 Maßnahmen (Kontakt- und Mobilitätseinschränkungen, Schließung von Kindertagesstätten und Schulen etc.) ergriffen, um die Ausbreitung von COVID-19 zu verhindern. Insbesondere zur Infektion, zum klinischen Verlauf und zur Infektiosität bei Kindern gibt es bislang wenig verlässliche Daten, die zur Entscheidung über die Wiedereröffnung von Kinderbetreuungseinrichtungen beitragen.

Kinder erkranken nach Infektion mit SARS-CoV-2 meist weniger schwer als Erwachsene, bei ihnen sind symptomlose oder symptomarme Verläufe häufiger [1].

Daten zur Prävalenz bei jüngeren Kindern werden insbesondere benötigt, um Aussagen zur Gefahr der Weitergabe der Infektion an ältere und vulnerable Personen, die im selben Haushalt leben, zu ermöglichen. Der Infektionsstatus bzgl. SARS-CoV-2 ist bei Kindern und deren Müttern oft kongruent. Die Übertragung der SARS-CoV-2-Infektion erfolgt hauptsächlich durch Erwachsene, insbesondere durch Haushaltskontakte [2]. Eine Studie hat gezeigt, dass dem Symptombeginn bei 7 infizierten Kindern eine nachgewiesene COVID-19-Infektion bei erwachsenen Familienmitgliedern vorausging [3]. In einer weiteren Studie fanden sich bei 28 von 31 infizierten Kindern weitere erkrankte Familienmitglieder [4].

Eine deutsche Studie [5] berichtet über einen deutlichen Anstieg des Infektionsrisikos für andere Familienmitglieder, wenn ein infiziertes Kind im Haushalt lebt. Somit kann die Bestimmung der Antikörper gegen SARS-CoV-2 bei Müttern indirekt Auskunft über den Infektionsstatus bei deren Kindern geben.

In Mecklenburg-Vorpommern wurde zwischen dem 3.3.2020 und dem 3.5.2020 bei insgesamt 699 Personen SARS-CoV-2 detektiert. Das entspricht einer Inzidenz von 43 Erkrankungen pro 100000 Einwohnern. Diese relativ niedrige Inzidenz kann u. a. erklärt werden durch den geringen internationalen Reiseverkehr nach Mecklenburg-Vorpommern zu Beginn der Pandemie, die geringe Zahl an Großveranstaltungen, den frühen Aufbau von Abstrichzentren zur PCR-Diagnostik, die rasche Aufklärung von Infektionsketten durch die Gesundheitsämter und das distanziertere Sozialverhalten der Bevölkerung z. B. bei Begrüßungen.

Da die Zahl der Neuinfektionen inzwischen stark abgenommen hat $(\triangleright$ Tab. 1$)$, stellt sich die Frage, wie viele Menschen die Infektion bereits überstanden haben und durch Antikörper geschützt sind.

Für den in dieser Studie verwendeten ELISA-Test zum Nachweis von SARS-CoV-2-Antikörpern (Euroimmun) wird in der Literatur eine hohe Sensitivität und Spezifität angegeben [6]. In unserer Studie wurden die positiven und grenzwertigen Antikörperbefunde des ELISA-Tests mit einem indirekten Immunfluoreszenztest (IIFT) als Bestätigungstest überprüft. Diese Antikörpertests zielen nicht darauf ab, virusneutralisierende Antikörper nachzuweisen.
Bei positivem Antikörpernachweis kann jedoch von einem Kontakt mit SARS-CoV-2 und einem gewissen Immunschutz ausgegangen werden, zumal mehrere Studien eine günstige Wirkung von antikörperhaltigem Rekonvaleszenten-Plasma auf den Verlauf von COVID-19 nahelegen [7].

In dieser Pilotstudie wurden 401 Mütter von Kindern im Alter von 1-10 Jahren als Sentinel untersucht, um die Prävalenz der Infektion mit SARS-CoV-2 auch bei Kindern abzuschätzen.

\section{Patienten, Material und Methoden}

Vom 20. bis 22.4.2020 hatten sich 401 Rostocker Mütter mit Kindern im Alter von 1-10 Jahren nach einem Zeitungsaufruf im Kompetenzzentrum für Klinische Studien der Universitätsmedizin Rostock zur Teilnahme an der Studie angemeldet. Nach dem Ausfüllen der Einverständniserklärung und des Fragebogens zu den Kindern, deren Unterbringung und den Risikofaktoren wurde ein Rachenabstrich (UTM Collection Swab Lot 1921668, Copan Italia S. p. a., Brescia, Italien) durchgeführt, Blut abgenommen und das daraus gewonnene Serum bei $-70^{\circ} \mathrm{C}$ aufbewahrt. Verlaufsseren von 10 stationären COVID-19-Patienten (Virus-RNA im Rachenabstrich positiv) unserer Klinik dienten als zusätzliche Positivkontrollen.

Die Ethikkommission an der Universitätsmedizin Rostock hat am 17.4.2020 ein positives Votum zu dieser Studie abgegeben (Registrier-Nummer: A2020-0090). Jede Studienteilnehmerin hat schriftlich ihr Einverständnis zur Teilnahme an dieser Studie erklärt.

Die Studie ist beim Deutschen Register Klinischer Studien (DRKS) unter der Nummer DRKS00 021614 erfasst.

\section{PCR-Virusnachweis}

SARS-CoV-2-Analysen von oropharyngealen Abstrichen wurden mit dem Allplex 2019-nCoV Assay (Ref. RP10 243X, Seegene Inc., Seoul, Korea) durchgeführt. Die Isolation der Nukleinsäuren wurde auf dem automatischen Extraktionssystem Microlab NIMBUS (Seegene) unter Verwendung des STARMag Universal Cartridge Kit (ref. 744 300.4.UC384, Seegene) durchgeführt. Die Amplifikations- und Detektionsschritte erfolgten auf einem CFX96 RealTime System (Bio-Rad Laboratories, California, USA) mit Bio-Rad CFX Manager IVD und 2019-nCov Viewer Software.

\section{Nachweis spezifischer Antikörper}

Die Seren wurden mit dem „Anti-SARS-CoV-2-ELISA (IgA)“ (EI 2606-9601 A) und dem „Anti-SARS-CoV-2-ELISA (IgG)“ (El 2606-9601 G) (Fa. Euroimmun, Lübeck, Deutschland) nach Herstellerangaben auf Antikörper gegen SARS-CoV-2 untersucht. Die Ergebnisse der Patientenproben wurden als Quotient aus dem Extinktionswert der Patientenprobe, dividiert durch den Extink- 
- Tab. 1 Gemeldete COVID-19-Fälle in Mecklenburg-Vorpommern nach Altersgruppe und Meldewoche (Stand: 04.05.2020).

\begin{tabular}{|c|c|c|c|c|c|c|c|c|c|c|}
\hline \multicolumn{11}{|c|}{ Meldewoche } \\
\hline Alter & 10 & 11 & 12 & 13 & 14 & 15 & 16 & 17 & 18 & gesamt \\
\hline $0-4$ & & & & & 3 & 1 & & 1 & & 5 \\
\hline $5-9$ & & & & 1 & 5 & 2 & & & & 8 \\
\hline $10-14$ & & 1 & & 4 & 3 & 1 & & 2 & 1 & 12 \\
\hline $15-19$ & & & 1 & 8 & 7 & 5 & & & 2 & 23 \\
\hline $20-24$ & & 4 & 13 & 13 & 10 & 8 & 5 & & 1 & 54 \\
\hline $25-29$ & & 2 & 22 & 22 & 6 & 5 & 6 & 1 & & 64 \\
\hline $30-34$ & & 1 & 19 & 14 & 9 & 4 & 2 & 1 & 1 & 51 \\
\hline $35-39$ & & 6 & 11 & 11 & 11 & 1 & 1 & 2 & & 43 \\
\hline $40-44$ & & 5 & 12 & 14 & 17 & 9 & & & 3 & 60 \\
\hline $45-49$ & 1 & 2 & 9 & 15 & 8 & 2 & 3 & 1 & 2 & 43 \\
\hline $50-54$ & 2 & 8 & 24 & 12 & 17 & 7 & 5 & 6 & 4 & 85 \\
\hline $55-59$ & & 3 & 15 & 14 & 12 & 4 & 3 & 1 & 5 & 57 \\
\hline $60-64$ & 2 & & 10 & 15 & 12 & 9 & 6 & 1 & 2 & 57 \\
\hline $65-69$ & & & 7 & 4 & 7 & 9 & 3 & 3 & 1 & 34 \\
\hline $70-74$ & & 1 & 3 & 6 & 5 & 2 & & & & 17 \\
\hline $75-79$ & 2 & & 2 & 8 & 13 & 6 & 1 & & & 32 \\
\hline $80+$ & & & 2 & 11 & 18 & 15 & 2 & 2 & 4 & 54 \\
\hline gesamt & 7 & 33 & 150 & 172 & 163 & 90 & 37 & 21 & 26 & 699 \\
\hline
\end{tabular}

tionswert einer mitgelieferten Kalibratorprobe, ausgedrückt. Quotienten unter 0,8 wurden als negativ, Werte ab 0,8, jedoch unter 1,1 wurden als grenzwertig und Quotienten ab 1,1 wurden als positiv befundet. Bei diesen Schwellenwerten werden nach Herstellerangaben keine Kreuzreaktionen mit anderen humanpathogenen Coronaviren (HCoV-229E, HCoV-OC43) gefunden. Positive oder grenzwertige Ergebnisse wurden durch Wiederholung der Untersuchung in einem unabhängigen Testansatz überprüft.

Die im ELISA-Test positiv oder grenzwertig getesteten Seren wurden zusätzlich im indirekten Immunfluoreszenztest (IIFT) zur Bestimmung von Antikörpern gegen SARS-CoV-2 untersucht [8] ( Abb. 1). Die Detektion der Serum-Antikörper gegen SARS-CoV-2 erfolgte mit anti-IgG-, IgA- und IgM-FITC-markierten Sekundärantikörpern (IgG: Fa. Sifin Cat. No. PA1107; IgA: Fa. Novex, Cat. No. A18 782; IgM: Fa. KPL Cat. No. 02-10-0) auf infizierten Aceton-Methanol-fixierten Vero-E6-Zellen (ATCC ${ }^{\circledR}$ CRL-1586) $[9,10]$. Das Virus war zuvor im BSL3-Labor des Bernhard-NochtInstituts für Tropenmedizin in Hamburg aus einer Patientenprobe isoliert und in Vero-E6-Zellen kultiviert worden. Beim Immunfluoreszenztest binden die spezifischen Antikörper an die Virusantigene innerhalb der infizierten und fixierten Zellen und ergeben ein charakteristisches Fluoreszenzmuster in den Zellen ( $\mathbf{A} \mathbf{A b} \mathbf{b} . \mathbf{1})$. Da bei Verdünnungsstufen unter 1:20 unspezifische oder nicht reproduzierbare Fluoreszenzen vorkommen können und es bei hohen Antikörperkonzentrationen oder bei Vorhandensein von Autoantikörpern zu Blockierungsphänomenen und falsch negati- ven Befunden kommen kann [11], wurden alle Serumproben mit einer Verdünnung von 1:10 bis 1:80 getestet. Titer unter 1:20 wurden als negativ bewertet [10].

Vom Landesamt für Gesundheit und Soziales in M-V wurden die Zahlen zur Altersverteilung der SARS-CoV-2-RNA-positiv getesteten Patienten in Mecklenburg-Vorpommern zur Verfügung gestellt.

\section{Ergebnisse}

Zwischen dem 22. und 29.4.2020 wurden 401 Mütter aus Rostock (Alter 36,9 \pm 4,9 Jahre) mit insgesamt 666 Kindern im Alter von 0-17 Jahren (mittleres Alter 5,2 $\pm 3,5$ Jahre) in die Studie eingeschlossen (ältere Kinder wurden nicht erfasst). Das entspricht durchschnittlich 1,66 Kinder pro teilnehmender Mutter. 182 Mütter $(45,4 \%)$ gaben an, mit 1 Kind im Haushalt zu leben, 179 (44,6\%) mit 2 Kindern, 34 (8,5\%) mit 3 Kindern und 6 (1,5\%) mit 4 Kindern.

Von den 34 Kindern bis zu 1 Jahr (5\% aller Kinder) wurden 33 zu Hause betreut und eins in einer Kita. 13 Kinder zwischen 1 und 4 Jahren wurden von einer Tagesmutter betreut. 396 Kinder im Alter von 1 bis 7 Jahren ( $59 \%$ aller Kinder, mittleres Alter 3,5 $\pm 1,6$ Jahre) wurden in einer von 58 der 90 Rostocker Kitas betreut. Von diesen 396 Kita-Kindern befanden sich 62 (16\%) nach dem 17.3.2020 in einer Kita-Notbetreuung. 173 Kinder (26\% aller Kinder, mittleres Alter 8,2 $\pm 1,5$ Jahre) besuchten 21 der insgesamt 


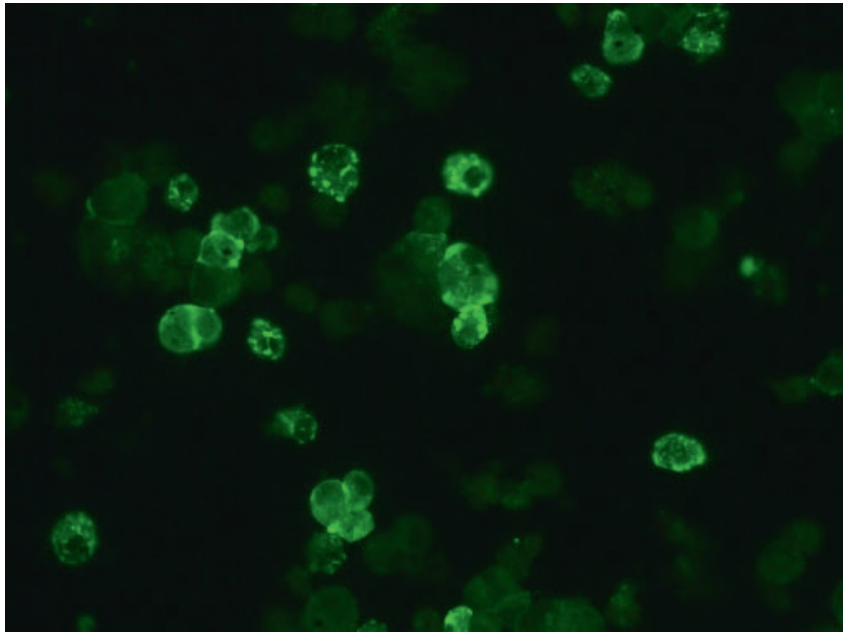

- Abb. 1 Indirekte Immunfluoreszenz mit anti-IgG-FITC-markierten Sekundärantikörpern: SARS-CoV-2-positive Vero-E6-Zellen mit grün fluoreszierenden intrazellulären granulären Strukturen.

29 Rostocker Grundschulen. Davon gingen 45 nachmittags in einen Hort, 20 auch noch nach dem 17.3.2020 in eine Notbetreuung (https://rathaus.rostock.de).

Keine der teilnehmenden Frauen war wissentlich an COVID-19 erkrankt. 172 der 401 Mütter waren nach dem 1.2.2020 außerhalb $M-V$ verreist. Sieben Frauen berichteten über einen Kontakt zu einer an COVID-19 erkrankten Person. Im genannten Zeitraum hatten 316 Frauen Erkältungssymptome, davon gaben 24 bei gezielter Befragung auch Geruchs- bzw. Geschmacksstörungen an. 100 (24,9\%) der 401 Mütter hatten eine tetravalente Grippeimpfung im Herbst 2019 erhalten.

Alle 401 Mütter waren in der PCR zum Nachweis von SARSCoV-2-RNA aus dem Rachenabstrich negativ.

Im IgG-ELISA-Test auf das Vorhandensein von SARS-CoV-2-spezifischen Antikörpern war ein Serum positiv. Im IgA-ELISA waren 11 Seren positiv und 3 Seren grenzwertig. Das Serum, das im IgG-ELISA positiv war, und die 14 Seren, die im IgA-ELISA positiv oder grenzwertig waren, wurden in der IIFT bzgl. IgG-, IgM- und IgA-Antikörper negativ getestet. Somit konnten in keinem der 401 Seren spezifische Antikörper gegen SARS-CoV-2 bestätigt werden.

In Mecklenburg-Vorpommern wurden zwischen der 10. und 18. Kalenderwoche (3.3. bis 3.5.2020) 699 SARS-CoV-2-Infektionen an die Gesundheitsämter gemeldet, davon 5 Fälle bei Kindern von 0-4 Jahren, 8 Fälle bei Kindern von 5-9 Jahren, 12 Fälle bei Kindern von 10-14 Jahren und 23 Fälle bei Jugendlichen von 15-19 Jahren. Bei den anderen Altersgruppen wurden zusammen 651 Fälle gemeldet. Das bedeutet, dass in Mecklenburg-Vorpommern ca. 0,7\% der Infektionen bei Kindern unter 4 Jahren und ca. 3,6\% der Infektionen bei Kindern unter 14 Jahren diagnostiziert wurden. SARS-CoV-2-positive Kinder unter 4 Jahren wurden erst ab dem 30.3.2020 gefunden, also 2 Wochen nach dem Schließen der Kinderbetreuungsstätten ( $\bullet$ Tab. 1).

\section{Diskussion}

Für die Schließung bzw. Wiedereröffnung von Kindergärten und Schulen während bzw. nach der COVID-19-Pandemie gibt es nur unzureichende Daten in der medizinischen Fachliteratur [10]. Wir haben in einer Pilotstudie bei 401 Müttern nach Virus-RNA im Rachenabstrich und nach spezifischen Antikörpern im Serum gesucht, um die Verbreitung des Virus bei Müttern als Sentinel für die Familien zu evaluieren. Gleichzeitig wurden die verwendeten Antikörpertests auf ihre Spezifität, d. h. den Nachweis falsch positiver Seren, bewertet. Die Sensitivität, d. h. den Nachweis falsch negativer Seren, konnten wir in dieser Studie nicht bewerten. Bei 10 stationären COVID-19-Patienten unserer Klinik konnten wir im Verlauf der Erkrankung allerdings IgG- und IgA-Antikörper im ELISA-Test nachweisen und mit dem IIFT für IgG, IgA und IgM bestätigen.

Auch wenn die Viruslast bei Kindern ähnlich hoch ist wie bei Erwachsenen [13], und bei symptomatischen Kindern unter einem Jahr sogar noch höher ist als bei älteren Kindern, haben Kleinkinder weniger schwere Erkrankungen als ältere Kinder [14].

In Mecklenburg-Vorpommern wurden auch deutlich weniger Infektionen bei Kindern als bei Erwachsenen gemeldet. Diese niedrige Infektionsrate bei Kindern deckt sich mit den Aussagen anderer Studien $[15,16]$. Die Daten einer weiteren Studie aus Shanghai und Wuhan legen nahe, dass die Empfänglichkeit für die Infektion mit SARS-CoV-2 bei Kindern unter 15 Jahren nur etwa ein Drittel so hoch ist wie bei 15- bis 65-Jährigen, und dass die Schließung von Schulen und Kindergärten trotzdem wahrscheinlich einen signifikanten Beitrag zur Verlangsamung der Infektionsausbreitung geleistet hat [18].

Einige andere Studien aus China treffen wiederum die Aussage, dass Kinder ähnlich empfänglich sind für die Infektion mit SARSCoV-2 wie Erwachsene [1, 17].

Die Infektiosität von mit SARS-CoV-2 infizierten Kindern unterliegt möglicherweise starken interindividuellen Schwankungen, wobei RNA-Viruslast nicht gleichbedeutend ist mit infektiösem Virus. So hat ein mit SARS-CoV-2 infizierter 9-jähriger Junge die Infektion trotz zahlreicher sozialer Kontakte nicht weitergegeben [19].

Unsere Ergebnisse unterstützen die Annahme, dass die Virusund Seroprävalenz gegen SARS-CoV-2 bei Müttern in Rostock sehr niedrig ist, und stehen mit der geringen Zahl der an COVID19 Erkrankten in der Stadt Rostock in Einklang. Die Aussagekraft dieser Studie zur Prävalenz der Infektion mit SARS-CoV-2 bei Müttern ist durch die relativ geringe Fallzahl ( $5 \%$ der Mütter mit schulpflichtigen Kindern in Rostock) limitiert. Weitere Studien zur Durchseuchung von Müttern werden helfen, die Prävalenz der Infektion mit SARS-CoV-2 in Familien genauer zu bestimmen. Da in dieser Studie durch die indirekte Immunfluoreszenz weder das positive Ergebnis des ELISA für $\mathrm{gGG}$, noch die 11 positiven und 3 grenzwertigen Ergebnisse des ELISA für I gA bestätigt wurden, empfehlen wir, positive SARS-CoV-2-Antikörperbefunde in den hier verwendeten ELISA-Tests mittels Bestätigungstests (z. B. indirekte Immunfluoreszenz) zu überprüfen. 


\section{KERNAUSSAGEN}

- Bei keiner von 401 Rostocker Müttern von Kindern zwischen 0 und 17 Jahren waren im April 2020 Antikörper gegen SARS-CoV-2 im Blut oder in der RNA im Rachenabstrich nachweisbar.

- In Mecklenburg-Vorpommern wurden bis 4.5.2020 bei Kindern unter 4 Jahren nur ca. 0,7\% und bei Kindern unter 14 Jahren nur ca. 3,6\% aller gemeldeten COVID-19-Infektionen diagnostiziert.

- Ein Nachweis von Serum-Antikörpern gegen SARS-CoV-2 im ELISA sollte mit einem Bestätigungstest (z. B. Immunfluoreszenz) überprüft werden.

Interessenkonflikt

Die Autorinnen/Autoren geben an, dass kein Interessenkonflikt besteht.

Danksagung

Wir danken Fr. K. Russow, Fr. Dr. M. Sombetzki, Fr. K. Wege, Fr. D. MichelLübs, Fr. C. Haprich, Fr. L. Peters, Fr. S. Pritsch, Fr. Dr. G. Brinschwitz, $\mathrm{Hr}$. C. Verbeek und Fr. E. Acksel, Universitätsmedizin Rostock, und Fr. Prof. G. Doblhammer, Max-Planck-Institut für Demografie, Rostock, und Prof. J. May und Prof. J. Schmidt-Chanasit, Bernhard-Nocht-Institut für Tropenmedizin Hamburg für die technische Unterstützung bei der Studie und die Hilfe bei der Erstellung des Manuskripts. Wir danken dem Ministerium für Wirtschaft, Arbeit und Gesundheit Mecklenburg-Vorpommern für die finanzielle Unterstützung dieser Studie. Wir bedanken uns auch bei der Fa. Seegene Deutschland GmbH, Düsseldorf, für die Bereitstellung der Allplex-2019-nCoV-Assay-Testreagenzien für diese Studien.

Literatur

[1] Dong $Y$, Mo X, Hu Y et al. Epidemiology of COVID-19 among children in China. Pediatrics 2020; 145: e2020-e0702. doi:10.1542/peds.2020-0702

[2] Choi SH, Kim HW, Kam JM et al. Epidemiology and clinical features of coronavirus disease 2019 in children. CEP 2020; 63: 125-132. doi:10.3345/cep.2020.00535

[3] Han YN, Feng ZW, Sun LN et al. A comparative-descriptive analysis of clinical characteristics in 2019-coronavirus-infected children and adults. J Med Virol 2020; 92: 1-7. doi:10.1002/jmv.25835

[4] Wang D, Ju XL, Xie F et al. Clinical analysis of 31 cases of 2019 novel coronavirus infection in children from six provinces (autonomous region) of northern China. Zhonghua Er Ke Za Zhi 2020; 58: 269-274. doi:10.3760/cma.j.cn112140-20200225-00138

[5] Streeck H, Schulte B, Kümmerer BM et al. Infection fatality rate of SARSCoV-2 infection in a German community with a super-spreading event. 04. Mai 2020. abgerufen am 19.05.2020. https://www.ukbonn.de/ C12582D3002FD21D/vwLookupDownloads/Streeck_et_al_Infection_ fatality_rate_of_SARS_CoV_2_infection2.pdf/\%24FILE/Streeck_et_al_ Infection_fatality_rate_of_SARS_CoV_2_infection2.pd
[6] Krüttgen A, Cornelissen CG, Dreher M et al. Comparison of four new commercial serologic assays for determination of SARS-CoV-2 IgG. Journal of Clinical Virology 2020; 128: 104394. doi:10.1016/j.jcv.2020. 104394

[7] Tamburello A, Marando M. Immunoglobulins or convalescent plasma to tackle COVID-19: buying time to save lives - current situation and perspectives. Swiss Med Wkly 2020; 150: w20264. doi:10.4414/ smw.2020.20264. eCollection 2020 Apr 20

[8] Wölfel R, Corman VM, Guggemos W et al. Virological assessment of hospitalized patients with COVID-19. Nature 2020; 581: 465-469. doi:10.1038/s41586-020-2196-x

[9] Kumar M, Mazur S, Ork BL et al. Inactivation and safety testing of Middle East Respiratory Syndrome Coronavirus. J Virol. Methods 2015; 223: 13-18

[10] Emmerich P, Thome-Bolduan C, Drosten C. Reverse ELISA for IgG and IgM antibodies to detect Lassa virus infections in Africa. J Clin Virol 2006; 37: 277-281. doi:10.1016/j.jcv.2006.08.015

[11] Gresner AM, Arndt T (Hrsg.). Lexikon der Medizinischen Laboratoriumsdiagnostik, Lexikon der Autoimmundiagnostik. 2. Auflage Springer-Verlag Heidelberg; 2013: 198-199

[12] Cowling B], Ali ST, Ng TWY et al. Impact assessment of non-pharmaceutical interventions against coronavirus disease 2019 and influenza in Hong Kong: an observational study. Lancet Public Health 2020; 5: e279-e288. doi:10.1016/S2468-2667(20)30090-6

[13] Jones TC, Mühlemann B, Veith T et al. An Analysis of SARS-CoV-2 viral load by patient age. 29.04.2020. heruntergeladen am 19.05.2020. https://virologie-ccm.charite.de/fileadmin/user_upload/microsites/ m_cc05/virologie-ccm/dateien_upload/Weitere_Dateien/analysisof-SARS-CoV-2-viral-load-by-patient-age-v2.pdf

[14] Zachariah P, Halabi KC, Johnson CL et al. Symptomatic Infants have Higher Nasopharyngeal SARS-CoV-2 Viral Loads but Less Severe Disease than Older Children. Clin Infect Dis 2020; 66: ciaa608. doi:10.1093/cid/ ciaa608

[15] Zimmermann P, Curtis N. Coronavirus Infections in Children Including COVID-19: An Overview of the Epidemiology, Clinical Features, Diagnosis, Treatment and Prevention Options in Children. The Pediatric Infectious Disease Journal 2020; 39: 355-368

[16] Mehta NS, Mytton OT, Mullins EWS et al. SARS-CoV-2 (COVID-19): What do we know about children? A systematic review. Clin Infect Dis 2020; 66: ciaa556. doi:10.1093/cid/ciaa556

[17] Bi Q, Wu Y, Mei S et al. Epidemiology and Transmission of COVID-19 in Shenzhen China: Analysis of 391 cases and 1.286 of their close contacts. Lancet Infect Dis 2020; 20: in press. pii: S1473-3099(20)30287-5. doi:10.1016/S1473-3099(20)30287

[18] Zhang J, Litvinova M, Liang Y et al. Changes in contact patterns shape the dynamics of the COVID-19 outbreak in China. Science 2020; 364: eabb8001. doi:10.1126/science.abb8001

[19] Danis K, Epaulard O, Bénet T et al. Cluster of Coronavirus Disease (COVID-19) in the French Alps, 2020. Clin Infect Dis 2020; 69: ciaa424 https://doi.org/10.1093/cid/ciaa424 